\title{
Progression of Cognitive Deficit in Older People with Mild Cognitive Impairment Treated with Cerebrolysin
}

\author{
Irina S. Boksha1, Svetlana I. Gavrilova², Igor V. Kolykhalov², Iana B. Fedorova², \\ Iaroslav B. Kalyn², Natalia D. Selezneva², Andrei V. Samorodov³, Serge N. Miasoedov ${ }^{4}$, \\ Elizabeta B. Mukaetova-Ladinska ${ }^{5}$ \\ ${ }^{1}$ Laboratory of Neurochemistry, Moscow, Russia \\ ${ }^{2}$ Department of Alzheimer's Disease and Related Disorders, Moscow, Russia \\ ${ }^{3}$ Department of Bioengineering and Medical Equipment and Systems, Moscow State Technical University, \\ Moscow, Russia \\ ${ }^{4}$ Laboratory of Biostatistics and Evidence Based Medicine, Mental Health Research Center of Russian Academy \\ of Medical Sciences, Moscow, Russia \\ ${ }^{5}$ Institute of Neurocience, Newcastle University Institute for Ageing, Newcastle University, Campus for Ageing \\ and Vitality, Newcastle upon Tyne, UK \\ Email: boksha irina@mail.ru
}

Received 1 September 2014; revised 16 October 2014; accepted 2 November 2014

Copyright (C) 2014 by authors and Scientific Research Publishing Inc.

This work is licensed under the Creative Commons Attribution International License (CC BY).

http://creativecommons.org/licenses/by/4.0/

(c) (i) Open Access

\section{Abstract}

Objectives: Although people with amnestic mild cognitive impairment (aMCI) benefit from cerebrolysin treatment, some still develop dementia. The aim of the current study was to identify most informative clinical assessment tests to predict the therapy efficacy in aMCI subjects treated with cerebrolysin. Methods: We studied patients with amnestic mild cognitive impairment (aMCI; $\mathbf{n}=$ 53) who had regular neurocognitive and clinical psychiatric assessments and were treated with cerebrolysin i.v. infusions $20 \times 30 \mathrm{ml}$ twice a year over three years period. Data were analyzed using non-parametric statistics, cluster and linear discriminant analyses. Results: Combined mathematical modeling enabled to predict cognitive decline from aMCI to dementia in the cerebrolysin-treated patients based on their initial neurocognitive assessment scores. We identified a "dementia risk group" with fast cognitive decline (i.e. low efficacy of the treatment). Lower baseline scores in the Mattis Dementia Rating Scale Memory subtest, Mini-Mental State Examination (MMSE), 10 word list immediate recall, and Frontal Assessment Battery tests when accompanied by higher depression score (Hamilton Depression Rating Scale) suggest poor prognosis for aMCI patients treated with cerebrolysin. Changes in scores on the MMSE, Boston naming test, Digit span forward, and Wechsler scale subtest "Categorical associations" during the treatment course are more characteristic for patients who convert to dementia than their initial scores. Conclusions: aMCI subjects treated with cerebrolysin with lower baseline cognitive functioning and subclinical depres- 
sion have poor prognosis in terms of converting to dementia. Changes in the MMSE, Boston naming test, Digit span forward, and Wechsler scale subtest "Categorical associations" scores during the treatment course are more informative to identify patients who will develop dementia than their initial scores.

\title{
Keywords
}

\author{
Mild Cognitive Impairment, Cerebrolysin, Dementia, Cluster Analysis, Linear Discriminant \\ Analysis
}

\section{Introduction}

A number of population studies have consistently reported that people with mild cognitive impairment (MCI) frequently develop Alzheimer's disease (AD) dementia [1]-[5]. The MCI syndrome, at least its amnestic type (aMCI), is now considered to represent an early (pre-clinical) stage of AD [6]. Clinical, psychopathological and neurocognitive studies have attempted to define a set of clinical signs which can serve as predictors of conversion to dementia in MCI subjects, and these include a set of tests used in the present study [7].

Despite numerous USA and European multi-center studies on identifying biological and clinical predictors of dementia conversion in MCI patients, little is known about how to detect MCI subjects at high risk to develop $\mathrm{AD}$ [8]. One way of addressing this is to use baseline and repeated screening data from long-term longitudinal studies on MCI. One such study is published by Gavrilova et al. [9]. This comparative controlled multi-center clinical study followed MCI subjects with no prominent vascular disorders who were on long-term therapy with cerebrolysin (regular four-week courses, twice a year) with an aim to prevent further cognitive decline and conversion to AD. In general, cerebrolysin demonstrated its high efficacy in prevention of cognitive decline and dementia development within three years in groups of 37 [10] and 55 [9] MCI patients.

The present study is a continuation of our work with further analysis of data obtained previously [9]. The main aim is to identify the most informative clinical attributes (e.g. clinical tests/scores) as predictors of MCI cognitive decline and whether they can distinguish "dementia risk group” among MCI patients enrolled in treatment trials. Since the frequency of ApoE $\varepsilon 4$ allele is both significantly higher in $\mathrm{AD}$ [11] and MCI subjects at high risk to convert to $\mathrm{AD}$ [9] [12], the present study also includes APOE genotyping data for all the studied MCI patients.

\section{Material and Methods}

\subsection{Subjects}

The study was approved by the Ethical Committee of the Mental Health Research Center (MHRC) of RAMS and was carried out in three Research Centers: Department of Alzheimer's Disease and Associated Disorders of MHRC RAMS (Moscow), Department of Mental and Neurological Diseases at the Military Medical Academy, St.-Petersburg, and Department of Neurology, Neurosurgery, and Psychiatry, Nizhny Novgorod State Medical Academy, Nizhny Novgorod, Russia. 53 patients in total were enrolled in the study, and assessed using an identical clinical battery. All participants signed an Informed Consent Form. Their median age was 68 years (53 - 83 years), 13 were males and 25 had ApoE $\varepsilon 4$ allele. Inclusion and exclusion criteria are listed in Table 1.

All subjects met the Petersen et al. [1] [13] criteria for aMCI (Memory problems, preferably corroborated by an informant; Impaired memory function for age and education; Preserved general cognitive function; Intact activities of daily living; Absence of dementia) and were regularly treated with cerebrolysin (Ebewe Pharma, Austria), a total number of $20 \times 30 \mathrm{ml}$ intravenous infusions, twice a year over a three year period (2007-2010).

All participants were clinically assessed with the following psychometric battery: Global Deterioration Scale (GDS) [14]; Clinical Dementia Rating (CDR) [15]; Hamilton Depression Rating Scale (HDRS) [16]; Modified Hachinski Ischemic Scale (MIS) [17]; Mini-Mental State Examination (MMSE) [18]; Mattis Dementia Rating Scale (MDRS) with subtests, incl. Reciprocal coordination, Graphomotor, Similarities, Memory [19]; Wechsler Scale Subtests (WAIS), incl. Acoustic associations, Categorical associations, Digit span forward (Repeat spoken 
Table 1. Inclusion and exclusion criteria for participants of the study.

\begin{tabular}{|c|c|}
\hline Inclusion criteria & Exclusion criteria \\
\hline 55 - 85 years people from both genders & Dementia diagnosis (according to DSM-IV, ICD-10 criteria) \\
\hline MMSE > 25/30 & $\begin{array}{l}\text { Neurological disorders (e.g. congenital and/or acquired metabolic encephalopathy, } \\
\text { toxic and drug-induced encephalopathy; Parkinson disease, multi-infarct dementia, } \\
\text { stroke, epilepsy, infectious diseases, demielinating and inherited neurodegenerative } \\
\text { disorders, traumatic brain injury) }\end{array}$ \\
\hline $\begin{array}{l}\text { Subjective memory complains confirmed by close } \\
\text { family member }\end{array}$ & Endocrine disorders, including decompensated diabetes \\
\hline Objective signs of memory problems & Neoplasm \\
\hline ADLs not overtly altered & HIV infection \\
\hline $\begin{array}{l}\text { Modified Hachinski Ischemic Scale (MIS) }<4 \text {, and } \\
\text { Hamilton Depression Rating Scale (HDRS) }<18 \text { at } \\
\text { baseline }\end{array}$ & Mental disorders \\
\hline $3^{\text {rd }}$ stage Global Deterioration Scale (GDS) & Alcoholism and/or drug dependence \\
\hline 0.5 score on Clinical Dementia Rating (CDR) & Drug induced or other intoxication \\
\hline No clinical diagnosis of dementia & Folate or vitamin B12 deficiency \\
\hline Signed Informed consent obtained & Systolic blood pressure $>180 \mathrm{mmHg}$, diastolic blood pressure $>95 \mathrm{mmHg}$ \\
\hline
\end{tabular}

list of digits in the same order-forward, Digit span backward; Word List Memory Task (10 word list learning and immediate recall; Delayed reproduction of 10 word list) [20]-[22]; Frontal Assessment Batterie (FAB) [23]; Clock Drawing Test (CDT) [24]-[26]) and Boston Naming Test (BNT) [27].

All participants underwent neuroimaging with magnetic resonance tomography to exclude underlying vascular pathology. APOE genotyping was done in the Laboratory of Molecular Genetics, MHRC RAMS. Genomic DNA was isolated from peripheral blood leukocytes using standard routine protocol [11] and APOE genotype determined with PCR [11].

\subsection{Statistics}

The analysis of data was done using the following statistical tests:

1. Non-parametric tests included Spearman rank-order correlation coefficients, Pairwise Wilcoxon Rank Sum Tests and Mann-Whitney U-test;

2. Cluster analysis was completed with Clust1 algorithm developed in the MHRC RAMS ([28]) and iterative cluster analysis. Clust1 algorithm was used for object clustering of 53 subjects (patients) against 14 attributes (i.e. scores in 14 neurocognitive tests after the treatment). Distinctive features of Clust1 algorithm are summarized as follows [28]: a) it uses data in a nominal scale based on number of observations for given value; b) it uses quantitative evaluation of an subject by attributes measured by different scales (nominal, ordinal, and ratio scale). The quantitative evaluation is calculated as a sum of quantitative measures of data belonging to distinct scales (e.g. nominal, ordinal, interval and ratio); c) it uses extensive method-determination of matrix of distances by analyses of all combinations of data; d) unlike most clustering methods, where using matrix of distances employs empiric evaluations for assignment of subjects to clusters, the Clust1 method renders the assignment of subjects to clusters based only on information structure of the matrix of distances without empiric criterions. Several clustering methods exist at present not involving usage of matrix of distances, but they do not employ combination of different scales of data; e) clustering by Clust1 algorithm, although not providing any model of a system under study, allows visualization of interrelationships between subjects (patients) and attributes (testing results, genotyping etc.).

3. Linear discriminant analysis (LDA).

LDA involves determination of weight coefficients to test results that are considered, where the value of final attribute is calculated for each patient:

$$
y=\sum_{i=1}^{N} a_{i} x_{i}
$$

where $N$-number of test results taken into account, $a_{i}$ - weight coefficient for $i$-th test, 
$x_{i}-$ numerical value of $i$-th test result.

The best distinction between two analyzed groups of participants (for instance, with/without dementia outcome) by the final attribute is usually established when the source data is normally distributed.

\section{Results}

An extensive database was created over the three year period containing a number of clinical parameters for all 53 participants, including age, gender, their APOE genotype, HDRS, MIS scores, as well as results of 14 neurocognitive tests (MMSE, FAB, CDT, Acoustic Associations, Categorical Associations, Digit span forward, Digit span backward, BNT, 10 word list immediate recall, Delayed word recall 10-Word (DWR), Reciprocal coordination, Graphomotor, Similarities, MDRS Memory subtest) prior and following their cerebrolysin treatment course. Three patients who completed the cerebrolysin treatment were diagnosed with AD.

\subsection{Correlation Analysis of Attributes Database}

In order to identify links between attributes, we performed a correlation analysis. All clinical attributes were significantly positively correlated (R from 0.41 to $0.63 ; p<0.002$ ), with the exception of HDRS and MDRS similarities subtests, that were negatively correlated $(\mathrm{R}=-0.4, p=0.002)$. None of the attributes (prior or after the treatment) was associated with participants' age.

\subsection{Cluster Analyses of Database}

Due to the high R correlations, we used clustering algorithms to define subgroup of MCI patients who have similar neuropsychological results as those patients who were diagnosed with dementia post-treatment. Cluster analysis, being applied to classification of subjects (patients) allows objective definition of similar subjects comprising homogenous subgroups from the total cohort of patients.

Clustering using Clust1 algorithm: Clustering was done by attributes, using the results from 14 neurocognitive tests after cerebrolysin treatment. We obtained four clusters using basic and modified Clust1, based on close correlations between analyzed attributes. One result was selected in which two clusters (K1, containing 34 patients, and K2, containing 19 patients) were obtained at the first level of clustering, with K2 containing all three MCI patients who converted to dementia. We, thus, refer to K2 as "unfavorable", or "at dementia risk" in relation to therapy efficacy, whereas K1 has a "favorable" outcome.

Participants' age was similar in both clusters, whereas more women (19/15) were found in the "unfavorable" K2 cluster. K2 cluster had worse performance (Mann-Whitney U-test, Bonferroni correction for multiple comparisons using strict criteria for significance of differences $p<0.006$ ) on following measures: MMSE, $p=$ 0.0015 ; FAB, $p=0.000003$; Categorical associations, $p=0.002$; 10 word list immediate recall, $p=0.0001$; Reciprocal coordination, $p=0.0003$; MDRS Memory subtest, $p=0.0004$; and BNT, $p=0.006$. Testing results for representatives of $\mathrm{K} 1$ and $\mathrm{K} 2$ clusters after the treatment are given in Table 2.

The attributes with a predictive value for MCI converting to dementia were explored in the data collected prior starting the treatment. The attributes (initial results of tests of patients comprising both K1 and K2), which differ significantly by Mann-Whitney U-test with Bonferroni' correction were: FAB, $p=0.0005$; 10 word list immediate recall, $p=0.002$; MDRS Memory subtest, $p=0.006$; and at tendency level-HDRS, $p=0.008$.

Definition of informative attributes - features of patients with dementia outcome: For the purpose of defining

Table 2. Results of tests after the treatment in K1 (favorable) and K2 (unfavorable) clusters.

\begin{tabular}{cccccc}
\hline Statistics & \multicolumn{3}{c}{ Tests } \\
\cline { 2 - 6 } & MMSE & FAB & $\begin{array}{c}\text { Categorical } \\
\text { associations }\end{array}$ & $\begin{array}{c}\text { BNT } \\
\begin{array}{c}10 \text { word list } \\
\text { immediate recall }\end{array}\end{array}$ MDRS Memory \\
subtest
\end{tabular}

MMSE, Mini-Mental State Examination; FAB, Frontal Assessment Batterie; BNT, Boston Naming Test; MDRS, Mattis Dementia Rating Scale. 
the attributes contributing to distinct treatment outcome, we divided the patient cohort into two subgroups: three patients with dementia outcome and other 50 patients who did not convert to dementia (Table 3). All three patients with dementia outcome were women with $\mathrm{ApoE} \varepsilon 4$ allele and had worse performance on their cognitive scores prior treatment, as well as post treatment, compared to other MCI subjects who did not convert to dementia (Table 3).

Iteration cluster analysis-Spearman distances: For the iteration cluster analysis, we used only the significantly different attributes (Table 3) that were also significantly different between K1 and K2 clusters (obtained with Clust1). The iteration cluster analysis, done with before the treatment attributes resulted in dendrogram using "Spearman distances" (which allows identification of three non-overlapping clusters of subjects/patients): the first cluster (K1a, 16 patients) contains two patients with dementia outcome, the second K2a (6 patients) contains one such patient (these two clusters may be regarded as "unfavorable” and "intermediate”, respectively) whereas the third cluster (K3a, 31 patients) does not contain any of the three patients with dementia outcome after the treatment; hence, it may be considered as the one with favorable treatment outcome.

k-Means clustering: Clustering of patients by k-means method (where $\mathrm{k}$ may be taken equal to three, based on the above results) and using Euclidean metric (Euclidean distance) gives three clusters K1b, K2b, K3b, with 20, 6 and 27 patients, respectively. The composition of these clusters did non-significantly differ from previous results; thus K1b contains one patient with dementia outcome and K2b contains two such patients.

Attributes obtained before, after the treatment, and measured only once (e.g. HDRS, MIS) were compared in the obtained clusters by Kruskal-Wallis and Chi-square methods. Similar results as those obtained previously were also confirmed with this method. Thus, the significant differences between the HDRS and the MIS scores were confirmed, as well as the differences in scores obtained with MMSE, FAB, 10 word list immediate recall, and MDRS Memory subtest before and after cerebrolysin treatment. Significant differences in scores in BNT and 10 word list delayed recall were observed after the treatment (data not shown).

The attribute "HDRS scores" significantly influenced the clustering results, because its exclusion from the attribute list (Table 3) results in only one cluster formation, consisting of all patients. This provides yet another indication of the HDRS importance in classifying our patients: the highest scores in this test were in the cluster with "unfavorable" treatment outcome (containing two patients with dementia), whereas the lowest HDRS scores in the cluster containing patients with "favorable" outcome (no dementia) only.

Although the cluster analysis identified favorable and unfavorable outcome groups pre- and post-treatment, none of the clustering results led to a clear separation of patients with dementia outcome from those without. However, using the applied clustering methods based on scores from clinical tests before the treatment, a risk group can be selected, containing MCI subjects who would deteriorate in their cognitive functioning during three-year period. Furthermore, the cluster analysis also identified certain neuropsychological attributes associated with a worse cognitive outcome, including higher scores high or low scores are individual scores relatively the maximum - peak of frequency distributions in the patient cohort, i.e. values which met with the highest frequency in the studied cohort of 53 patients in HDRS and lower scores in MMSE, MDRS "Memory" subtest,

Table 3. Results of comparison (Mann-Whitney U-test) between three patients with dementia outcome and others (50 patients of cohort) by attributes before the treatment and changes in scores in tests (initial-final).

\begin{tabular}{ccc}
\hline Tests & Test scores before the treatment Z & Changes in scores Z \\
\hline MDRS Memory subtest & $\mathbf{2 . 5 ^ { \mathrm { a } }}$ & $\mathbf{1 . 1}^{\mathrm{b}}$ \\
HDRS & $\mathbf{2 . 0}$ & $\mathbf{1 . 9}$ \\
MMSE & $\mathbf{1 . 7}$ & 0.9 \\
10 Word list immediate recall & $\mathbf{1 . 7}$ & $\mathbf{2 . 8}$ \\
BNT & 1.6 & $\mathbf{2 . 5}$ \\
Digit span forward & 0.5 & $\mathbf{1 . 9}$ \\
\hline
\end{tabular}

${ }^{\mathrm{a}}$ The first value (the second column in the table) refers to difference between two groups in their resulting tests before the treatment; ${ }^{\mathrm{b}}$ The second value (the third column in the table) — the difference between these groups in changes of scores, i.e. difference between the numerical values of test scores before and after the treatment. Those attributes for which $Z>1.64$, i.e. the difference between the groups in these attributes is significant with probability of $90 \%$, are given in bold. MDRS, Mattis Dementia Rating Scale; HDRS, Hamilton Depression Rating Scale; MMSE, Mini-Mental State Examination; BNT, Boston Naming Test. 
as well as 10 word list immediate recall and FAB, similar to those observed in MCI subjects with dementia outcome.

\subsection{Linear Discriminant Analysis}

We applied linear discriminant analysis (LDA) to clinical tests' scores to test the possibility of dividing the patients into two groups, patients with $(n=3)$, and without dementia $(n=50)$ outcome. Since there are two groups, the result of LDA is a mathematical transformation of scorings, representing their linear combination with the outcome of one numerical attribute. Since the group of patients with dementia outcome contains only three patients, the normality validation of the source data distribution for this group is not possible. The LDA was based on attributes selected as follows.

A. Linear separability of patient groups by all testing results as the source data. Values of weight coefficients were obtained using scores in 14 tests before the treatment (initial), and changes in testing results $(\Delta$, i.e. afterminus before the treatment) (Figure 1(a)). The left column in the histogram is presented by three patients with dementia outcome. Thus, the dataset (14 tests) allows clear separation of subjects with dementia from others. This suggests that the whole dataset, when the three attributes that were measured once (e.g. ApoE 4 genotype, scores in MIS and HDRS) are excluded, is sufficient for this analysis.

B. Linear separability of patient groups by changes in testing results during the treatment, taking into account three "single-measured" tests. The source data included results of three "single-measured" tests and 14 differences $(\Delta)$ between testing results after the treatment and initial testing results (Figure 1(b)). The left column

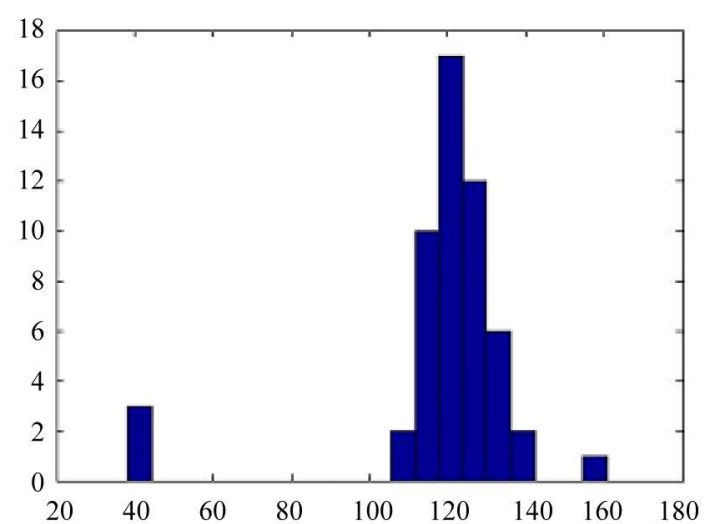

(a)

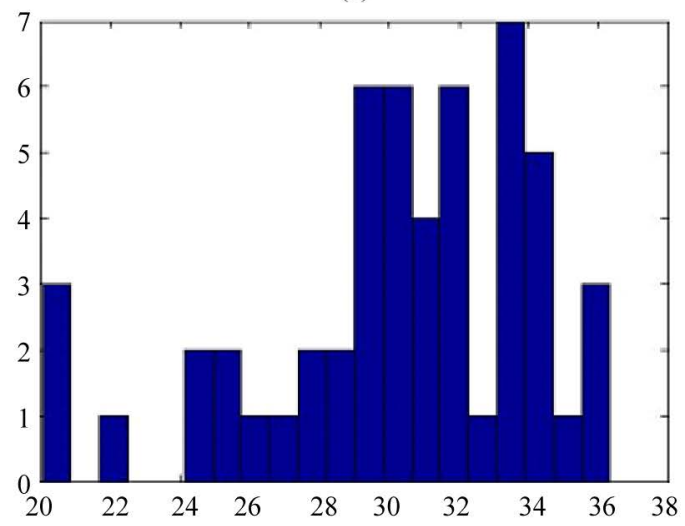

(c)

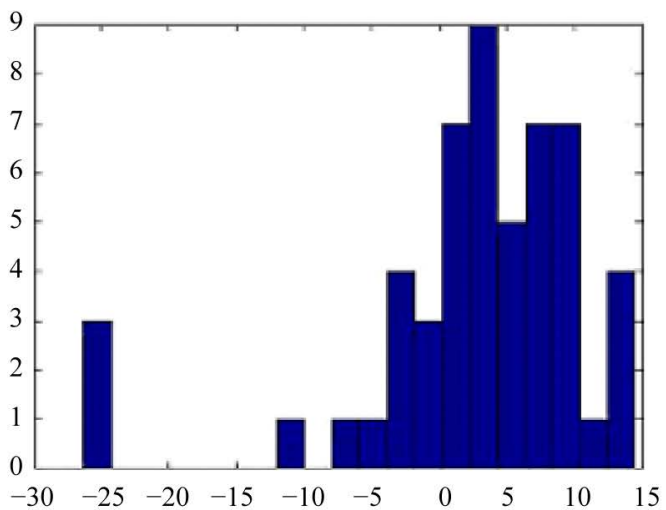

(b)

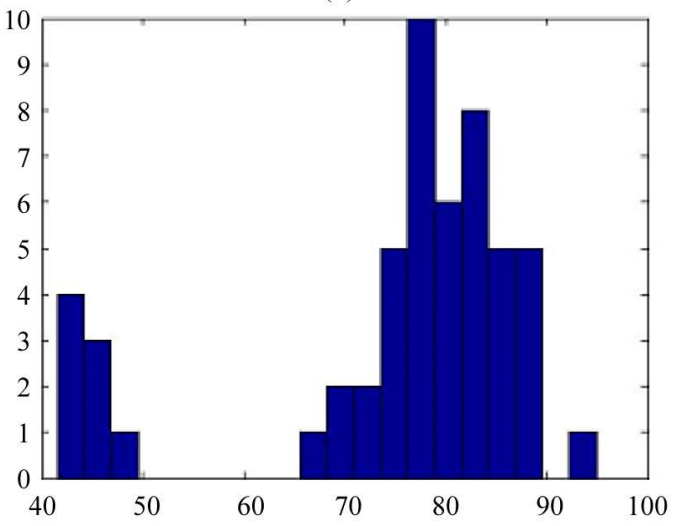

(d)

Figure 1. Histograms of patient distribution by the obtained numerical attribute: values of weight coefficients obtained when using as the source data: (a) Before the treatment (initial) and changes in testing results $(\Delta$, i.e. after minus before the treatment); (b) Changes $(\Delta)$ in testing results during the treatment, taking into account three "single-measured" tests; (c) Before the treatment (initial) testing results; (d) Before the treatment (initial) testing results with the risk group definition. 
represents the three patients with dementia outcome; note, one patient (presented by the second column from left) is separated from the rest patients.

C. Linear separability of two patients' groups (3 with dementia and others) by attributes before the treatment can be used to predict the treatment outcome (see Weight coefficients in Table 4), but in this case LDA is not as powerful to separate clearly the two distinct groups of patients (Figure 1(c)).

D. LDA of source data before the treatment with the risk group definition provides a reasonable differentiation between the groups (Figure $1(\mathrm{~d})$ ), the first ("risk group") contains the three patients with dementia outcome and also additional patients $(\mathrm{n}=5)$ with similar attributes.

The findings from the LDA analysis can be summarized as follows:

1) When the source data is the set of all 14 twice measured attributes, LDA allows separation of group consisting of three patients with dementia outcome from others (50 patients) (Figure 1(a)).

2) Changes in tests' scorings are more informative than scorings before the treatment for separation of patients with dementia outcome, but LDA enables separation of three patients with dementia outcome from others in the both cases (Figure 1(a) and Figure 1(b)).

3) When using only 14 scorings before the treatment for classification of patients, it is reasonable to separate at "risk group" containing, together with patients with dementia outcome, a few patients the most closely located to them by attributes (scores), but without dementia outcome (Figure 1(c)). When using all 17 tests (14 scores before the treatment and 3 single measured tests) the best results for the separation of groups correspond to risk group consisting of eight patients: three patients with dementia outcome plus five without dementia outcome (Figure 1(d)). The value of linear combination of scores in 17 tests less than the threshold value (55) corresponds to $37.5 \%$ probability of dementia development in individual patient.

\section{Discussion}

The MCI syndrome is heterogeneous and it is still unknown what clinical symptoms and measures predict poor outcome (e.g. conversion to dementia) [1] [2] [5] [7] [8] [29]-[32]. Detailed studies of neurocognitive functions

\begin{tabular}{lc} 
Table 4. Weight coefficients for testing results before the treatment. \\
\hline Genotyping & Weight coefficient \\
MIS & 3.31 \\
HDRS & -0.18 \\
MMSE initial & -0.44 \\
FAB initial & -0.13 \\
CDT initial & 0.03 \\
Acoustic Associations initial & -0.39 \\
Categorical Associations initial & -0.28 \\
Digit span forward initial & -0.36 \\
Digit span backward initial & 0.02 \\
BNT initial & 0.96 \\
10 word list immediate recall initial & 0.05 \\
Delayed word recall 10-Word (DWR) initial & -0.12 \\
Reciprocal coordination initial & 0.39 \\
Graphomotor initial & 0.89 \\
Similarities initial & 0.07 \\
MDRS Memory subtest initial & -0.75 \\
\hline
\end{tabular}

MIS, Modified Hachinski Ischemic Scale; MDRS, Mattis Dementia Rating Scale; HDRS, Hamilton Depression Rating Scale; MMSE, Mini-Mental State Examination; BNT, Boston Naming Test; CDT, Clock Drawing Test; DWR, Delayed word recall; FAB, Frontal Assessment Batterie. 
have identified "episodic memory block" and cued recall to be a prodromal state of AD in MCI subjects [32][35]. In line with previously described link between $\mathrm{AD}$ development and ApoE $\varepsilon 4$ allele [36], we confirm a higher risk of $\mathrm{AD}$ conversion in $\mathrm{APOE} \varepsilon 4$ allele aMCI careers, who also have significantly lower scores on a number of neurocognitive tests, including MMSE, FAB, CDT and WAIS “Acoustic Association” subscale test, despite being treated with cerebrolysin, a neurotropic that targets the size and number of dendrites and neurons. Furthermore, in the current study we use a novel clustering tool to aid the analysis of the complex clinical data that can find use in future clinical studies to predict the prognosis and outcomes in aMCI subjects.

Previous studies have similarly attempted to distinguish MCI subtypes and identify clinical and biomarker variables to predict MCI subjects at high risk to convert to dementia. Thus, "multifunctional" and "monofunctional" subtypes of amnestic MCI type (aMCI) [30] were separated on the basis of neurocognitive characteristics. The multifunctional aMCI type had a more rapid transition to dementia within 5 years of observation compared to the monofunctional aMCI; similarly, auditory sensor potentials differ in patients with various aMCI subtypes, and these differences are associated with various rates of cognitive decline progression and dementia development [4] [37].

Cerebrolysin is considered to be an effective therapy directed to prevent further cognitive decline in people suffering from dementia, MCI, as well as older people without cognitive impairment [9] [10] [38]-[41]. However, the therapeutic effect of cerebrolysin (e.g. halting cognitive decline) especially in MCI subjects, appears not to be uniform, with a small number still progressing to overt dementia. This probably reflects the heterogeneity of the MCI syndrome that is further confirmed with the poorer neurocognitive profile at baseline [9]. This is not surprising and provides further support of mono- and multifunctional subtypes of aMCI being of different biological origin with different probability of dementia outcome [5].

In the present work we have focused on parameter(s), which distinguish patients with distinct response profile to cerebrolysin treatment e.g. patients with stable and improved cognitive functioning (favorable subgroup) from those with aggravation of their cognitive mental functions (unfavorable treatment outcome). We did not have pre-set criteria for "unfavorable" and "favorable" outcomes, and their distinction was a result of extensive cluster analysis recognized as an informative method to classify subjects (patients and/or surveyed individuals) and attributes (signs, i.e. neurocognitive and psychometric results, questionnaires, demographic data and results from biological studies) from databases, and has been used in both psychiatric and neuropsychological studies [28].

In the present study, cluster analysis helped distinguish homogenous subgroups of patients following their anti-dementia treatment, taking into account their pre-treatment neuropsychological differences. This novel approach has enabled us to identify the most informative neurocognitive predictors of unfavorable outcome in aMCI subjects treated with cerebrolysin who further progressed to dementia. Furthermore, this analysis was successfully based on using the results from neurocognitive tests before the treatment was initiated. However, we have to highlight that not all eight patients within this subgroup received the dementia diagnosis (only three persons) after three years of treatment. This suggests that there are additional parameters (e.g. biochemical, neuroradiological etc.) that influence our model, and they may influence identifying subjects with highest dementia risk among the "risk group". We also cannot exclude the possibility that subjects in the risk group ("unfavorable" clusters) could have progressed to dementia if they have been followed longer.

The linear discriminant analysis (LDA) was helpful to separate the "risk group" (characterized by subsequent cognitive decline and unfavorable outcome following treatment) based on test results prior the treatment course. This is an important finding, since it can be used to determine the prognosis of the treatment outcomes in future clinical trials in aMCI subjects. However, this statistical method cannot be used to rank attributes (signs) and decrease the number of tests necessary for the calculation. Longer longitudinal studies on larger patients cohorts with inclusion of additional biological attributes (e.g. neuroimaging, CSF biomarkers, EEG etc.), in addition to APOE genotyping, would enhance the probability when making projections of dementia development for every patient with aMCI and develop differential individual approach to preventive AD therapy with cerebrolysin.

\section{Conclusions}

- The most informative tests used before commencing the treatment with cerebrolysin to predict progressive cognitive decline are: MDRS Memory subtest, HDRS, MMSE, Word List Memory Task (10 word list learning and immediate recall). Lower scores on these tests at baseline, as well as on FAB, and relatively higher scores in HDRS argue for unfavorable prognosis for aMCI patients treated with cerebrolysin. 
- When classifying patients using only their scores before the treatment, there is a reasonable separation of the "risk group", consisting of patients with dementia outcome alongside with those aMCI patients who have similarly lower cognitive scores, but who are not diagnosed with dementia following their 3 years treatment with cerebrolysin. When scores from all 17 tests (14 tests before the treatment and three single-measured tests) are used, the best group separation using the LDA corresponds to risk group of eight patients (three with dementia outcome and five with non-dementia outcome). The value of linear combination of scorings in 17 tests less than 55 points corresponds to $37.5 \%$ probability of dementia outcome.

- Changes in neurocognitive scores during the treatment course are more informative to identify aMCI patients who develop dementia than baseline scores obtained prior starting the treatment, with the most informative changes in MMSE, BNT, Digit span forward, and WAIS subtest "Categorical associations" scores.

\section{Acknowledgements}

We would like to thank all participants in the study and their care givers. EBM-L was supported by a grant from Alzheimer Society (London, UK).

\section{References}

[1] Petersen, R.C., Smith, G.E., Waring, S.C., Ivnik, R.J., Kokmen, E. and Tangelos, E.G. (1997) Aging, Memory, and Mild Cognitive Impairment. International Psychogeriatrics, 9, 65-69. http://dx.doi.org/10.1017/S1041610297004717

[2] Petersen, R.C., Smith, G.E., Waring, S.C., Ivnik, R.J., Tangalos, E.G. and Kokmen, E. (1999) Mild Cognitive Impairment: Clinical Characterization and Outcome. Archives of Neurology, 56, 303-308. http://dx.doi.org/10.1001/archneur.56.3.303

[3] Petersen, R.C. (2004) Mild Cognitive Impairment as a Diagnostic Entity. Journal of Internal Medicine, 256, $183-194$. http://dx.doi.org/10.1111/j.1365-2796.2004.01388.x

[4] Golob, E.J., Johnson, J.K. and Starr, A. (2002) Auditory Event-Related Potentials during Target Detection Are Abnormal in Mild Cognitive Impairment. Clinical Neurophysiology, 113, 151-161. http://dx.doi.org/10.1016/S1388-2457(01)00713-1

[5] Golob, E.J., Irimajiri, R. and Starr, A. (2007) Auditory Cortical Activity in Amnestic Mild Cognitive Impairment: Relationship to Subtype and Conversion to Dementia. Brain, 130, 740-752. http://dx.doi.org/10.0.4.69/brain/awl375

[6] Schmidtke, K. and Hermeneit, S. (2008) High Rate of Conversion to Alzheimer's Disease in a Cohort of Amnestic MCI Patients. International Psychogeriatrics, 20, 96-108. http://dx.doi.org/10.1017/S1041610207005509

[7] Gavrilova, S.I., Fedorova, I.B., Roshchina, I.F. and Korovaĭtseva, G.I. (2007) Prognosis of the Syndrome of Mild Cognitive Impairment: The Data of the Biennial Follow-Up Study. Zh Nevrol Psikhiatr Im S S Korsakova, 107, 4-10.

[8] Vemuri, P., Wiste, H.J., Weigand, S.D., Knopman, D.S., Trojanowski, J.Q., et al. (2010) Serial MRI and CSF Biomarkers in Normal Aging, MCI, and AD. Neurology, 75, 143-151. http://dx.doi.org/10.1212/WNL.0b013e3181e7ca82

[9] Gavrilova, S.I., Kolykhalov, I.V., Fedorova, I.B., Selezneva, N.D., Kalyn, I.B., Roshchina, I.F., et al. (2010) Possibilities of Preventive Treatment of Alzheimer's Disease: Results of the 3-Year Open Prospective Comparative Study on Efficacy and Safety of the Course Therapy with Cerebrolysin and Cavinton in Elderly Patients with the Syndrome of Mild Cognitive Impairment. Zh Nevrol Psikhiatr Im S S Korsakova, 110, 62-69.

[10] Gavrilova, S.I., Fedorova, Y.B., Roshchina, I.F. and Korovaitseva, G.I. (2008) Prognosis of Mild Cognitive Impairment Syndrome: Data from a Two-Year Clinical Follow-Up Study. Neuroscience and Behavioral Physiology, 38, 129134. http://dx.doi.org/10.1007/s11055-008-0018-y

[11] Korovaĭtseva, G.I., Shcherbatykh, T.V., Selezneva, N.V., Gavrilova, S.I., Golimbet, V.E., Voskresenskaia, N.I. and Rogaev, E.I. (2001) Genetic Association between the Apolipoprotein E (ApoE) Gene Alleles and Various Forms of Alzheimer's Disease. Genetika, 37, 529-535. (Article in Russian)

[12] Caselli, R.J., Dueck, A.C., Osborne, D., Sabbagh, M.N., Connor, D.J., Ahern, G.L., et al. (2009) Longitudinal Modeling of Age-Related Memory Decline and the APOE $\varepsilon 4$ Effect. New England Journal of Medicine, 361, 255-263. http://dx.doi.org/10.1056/NEJMoa0809437

[13] Petersen, R.C., Doody, R., Kurz, A., Mohs, R.C., Morris, J.C., Rabins, P.V., et al. (2001) Current Concepts in Mild Cognitive Impairment. JAMA Neurology, 58, 1985-1992. http://dx.doi.org/10.1001/archneur.58.12.1985

[14] Reisberg, B., Ferris, S.H., de Leon, M.J. and Crook, T. (1988) Global Deterioration Scale (GDS). Psychopharmacology Bulletin, 24, 661-663.

[15] Morris, J.C. (1993) The Clinical Dementia Rating (CDR): Current Version and Scoring Rules. Neurology, 43, 2412. http://dx.doi.org/10.1212/WNL.43.11.2412-a 
[16] Hamilton, M. (1960) A Rating Scale for Depression. Journal of Neurology, Neurosurgery \& Psychiatry, 23, 56-62. http://dx.doi.org/10.1136/jnnp.23.1.56

[17] Hachinski, V.C. (1978) Cerebral Blood Flow Differentiation of Alzheimer's Disease from Multi-Infarct Dementia. In: Katzman, R., Terry, R.D. and Bick, K.L., Eds., Alzheimer's Disease: Senile Dementia and Related Disorder, Raven Press, New York, 97-104.

[18] Folstein, M.F., Folstein, S.E. and McHugh, P.R. (1975) 'Mini-Mental State'. A Practical Method for Grading the Cognitive State of Patients for the Clinician. Journal of Psychiatric Research, 12, 189-198. http://dx.doi.org/10.1016/0022-3956(75)90026-6

[19] Mattis, S. (1976) Mental Status Examination for Organic Mental Syndrome in the Elderly Patient. In: Bellack, L. and Karusu, T.B., Eds., Geriatric Psychiatry, Grune \& Stratton, New York, 77-121.

[20] Wechsler, D. (1981) Wechsler Adult Intelligence Scale-Revised. Psychological Corporation, New York.

[21] Collie, A., Myers, C., Schnirman, G., Wood, S. and Maruff, P. (2002) Selectively Impaired Associative Learning in Older People with Cognitive Decline. Journal of Cognitive Neuroscience, 14, 484-492. http://dx.doi.org/10.1162/089892902317361994

[22] Takayama, Y. (2010) A Delayed Recall Battery as a Sensitive Screening for Mild Cognitive Impairment: Follow-Up Study of Memory Clinic Patients after 10 Years. Journal of Medical and Dental Sciences, 57, 177-184.

[23] Dubois, B., Slachevsky, A., Litvan, I. and Pillon, B. (2000) The FAB: A Frontal Assessment Battery at Bedside. Neurology, 55, 1621-1626. http://dx.doi.org/10.1212/WNL.55.11.1621

[24] Brodaty, H., Gresham, M. and Luscombe, G. (1997) The Prince Henry Hospital Dementia Caregivers’ Training Programme. International Journal of Geriatric Psychiatry, 12, 183-192. http://dx.doi.org/10.1002/(SICI)1099-1166(199702)12:2<183::AID-GPS584>3.0.CO;2-J

[25] Shulman, K.I., Shedletsky, R. and Silver, I.L. (1986) The Challenge of Time: Clock-Drawing and Cognitive Function in the Elderly. International Journal of Geriatric Psychiatry, 1, 135-140. http://dx.doi.org/10.1002/gps.930010209

[26] Sunderland, T., Hill, J.L., Mellow, A.M., Lawlor, B.A., Gundersheimer, J., Newhouse, P.A. and Grafman, J.H. (1989) Clock Drawing in Alzheimer's Disease: A Novel Measure of Dementia Severity. Journal of the American Geriatrics Society, 37, 725-729.

[27] Kaplan, E.F., Goodglass, H. and Weintraub, S. (1983) The Boston Naming Test. 2nd Edition, Lea \& Febiger, Philadelphia.

[28] Sudakov, S.A. (2010) Cluster Analysis in Psychiatry and Clinical Psychology: A Guide. [In Russian] Medical Information Agency, Moscow.

[29] Morris, J.C., Storandt, M., Miller, J.P., McKeel, D.W., Price, J.L., Rubin, E.H. and Berg, L. (2001) Mild Cognitive Impairment Represents Early-Stage Alzheimer Disease. JAMA Neurology, 58, 397-405. http://dx.doi.org/10.1001/archneur.58.3.397

[30] Winblad, B., Palmer, K., Kivipelto, M., Jelic, V., Fratiglioni, L., Wahlund, L.O., et al. (2004) Mild Cognitive Impairment-Beyond Controversies, towards a Consensus: Report of the International Working Group on Mild Cognitive Impairment. Journal of Internal Medicine, 256, 240-246. http://dx.doi.org/10.1111/j.1365-2796.2004.01380.x

[31] De Jager, C.A., Milwain, E. and Budge, M. (2002) Early Detection of Isolated Memory Deficits in the Elderly: The Need for More Sensitive Neuropsychological Tests. Psychological Medicine, 32, 483-491. http://dx.doi.org/10.1017/S003329170200524X

[32] De Jager, C.A., Schrijnemaekers, A.C., Honey, T.E. and Budge, M.M. (2009) Detection of MCI in the Clinic: Evaluation of the Sensitivity and Specificity of a Computerized Test Battery, the Hopkins Verbal Learning Test and the MMSE. Age and Ageing, 38, 455-460. http://dx.doi.org/10.1093/ageing/afp068

[33] Elias, M.F., Beiser, A., Wolf, P.A., Au, R., White, R.F. and D’Agostino, R.B. (2000) The Preclinical Phase of Alzheimer Disease: A 22-Year Prospective Study of the Framingham Cohort. JAMA Neurology, 57, 808-813. http://dx.doi.org/10.1001/archneur.57.6.808

[34] Schrijnemaekers, A.M., de Jager, C.A., Hogervorst, E. and Budge, M.M. (2006) Cases with Mild Cognitive Impairment and Alzheimer's Disease Fail to Benefit from Repeated Exposure to Episodic Memory Tests as Compared with Controls. Journal of Clinical and Experimental Neuropsychology, 28, 438-455. http://dx.doi.org/10.1080/13803390590935462

[35] De Jager, C.A. (2004) Changes over Time in Memory, Processing Speed and Clock Drawing Tests Help to Discriminate between Vascular Cognitive Impairment, Mild Cognitive Impairment and Alzheimer's Disease. Neurological Research, 26, 481-487. http://dx.doi.org/10.1179/016164104225016209

[36] Roses, A.D., Saunders, A.M., Corder, E.H., Pericak-Vance, M.A., Han, S.H., Einstein, G., et al. (1995) Influence of the Susceptibility Genes Apolipoprotein E-Epsilon 4 and Apolipoprotein E-Epsilon 2 on the Rate of Disease Expres- 
sivity of Late-Onset Alzheimer's Disease. Arzneimittel-Forschung, 45, 413-417.

[37] Narasimhalu, K., Ang, S., De Silva, D.A., Wong, M.C., Chang, H.M., Chia, K.S., et al. (2009) Severity of CIND and MCI Predict Incidence of Dementia in an Ischemic Stroke Cohort. Neurology, 73, 1866-1872. http://dx.doi.org/10.1212/WNL.0b013e3181c3fcb7

[38] Alvarez, X.A., Lombardi, V.R., Corzo, L., Pérez, P., Pichel, V., Laredo, M., et al. (2000) Oral Cerebrolysin Enhances Brain Alpha Activity and Improves Cognitive Performance in Elderly Control Subjects. Journal of Neural Transmission. Supplementum, 59, 315-328.

[39] Alvarez, X.A., Cacabelos, R., Laredo, M., Couceiro, V., Sampedro, C., Varela, M., et al. (2006) A 24-Week, Double-Blind, Placebo-Controlled Study of Three Dosages of Cerebrolysin in Patients with Mild to Moderate Alzheimer's Disease. European Journal of Neurology, 13, 43-54. http://dx.doi.org/10.1111/j.1468-1331.2006.01222.x

[40] Chen, N., Yang, M., Guo, J., Zhou, M., Zhu, C. and He, L. (2013) Cerebrolysin for Vascular Dementia. Cochrane Database of Systematic Reviews, 1, CD008900. http://dx.doi.org/10.1002/14651858.CD008900

[41] Damulin, I.V., Koberskaia, N.N. and Mkhitarian, E.A. (2007) The Effect of Cerebrolysin on Moderate Cognitive Impairment in Cerebral Vascular Insufficiency (a Clinical and Electrophysiological Study). Zhurnal Nevrologii i Psikhiatrii Imeni S.S. Korsakova, 107, 32-38. (Article in Russian)

\section{Abbreviations}

AD, Alzheimer's disease;

BNT, Boston Naming Test;

CDR, Clinical Dementia Rating;

CDT, Clock Drawing Test;

DWR, Delayed word recall;

FAB, Frontal Assessment Batterie;

GDS, Global Deterioration Scale;

HDRS, Hamilton Depression Rating Scale;

LDA, Linear discriminant analysis;

MCI, Mild Cognitive Impairment;

MDRS, Mattis Dementia Rating Scale;

MHRC, Mental Health Research Center;

MIS, Modified Hachinski Ischemic Scale;

MMSE, Mini-Mental State Examination;

WAIS, Wechsler Scale Subtests 
Scientific Research Publishing (SCIRP) is one of the largest Open Access journal publishers. It is currently publishing more than 200 open access, online, peer-reviewed journals covering a wide range of academic disciplines. SCIRP serves the worldwide academic communities and contributes to the progress and application of science with its publication.

Other selected journals from SCIRP are listed as below. Submit your manuscript to us via either submit@scirp.org or Online Submission Portal.
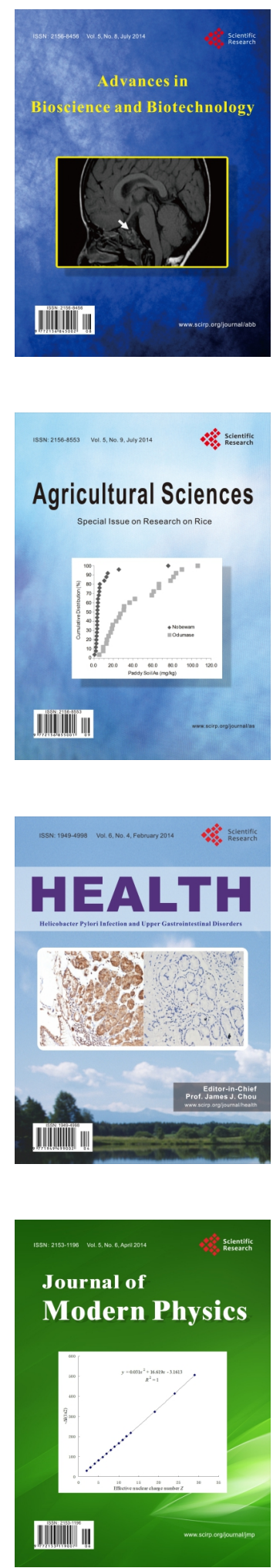
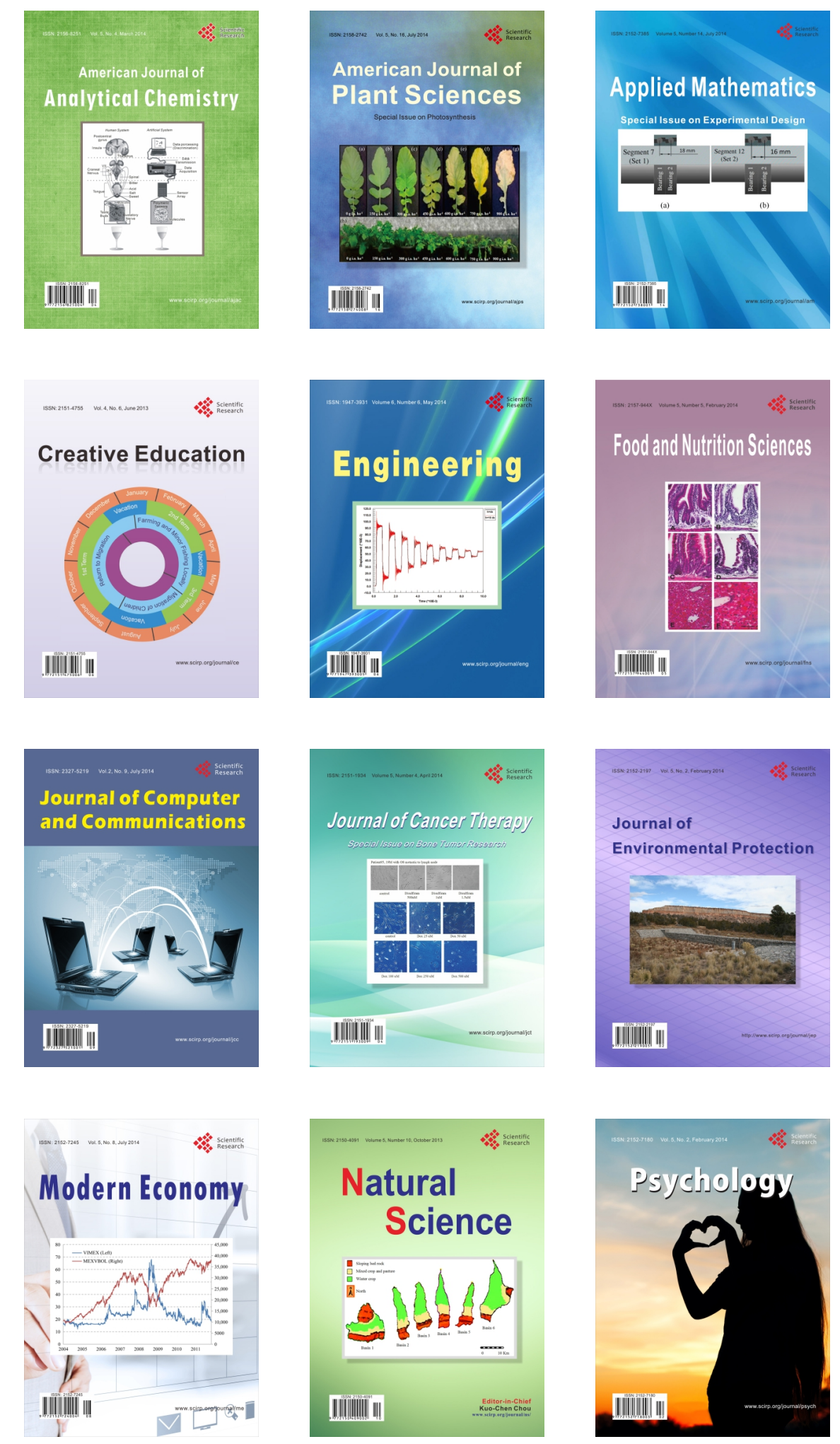